\title{
ANALOGUE HAWKING EFFECT: BEC AND SURFACE WAVES
}

\author{
F. BELGIORNO ${ }^{1,2,3}$, S.L. CACCIATORI ${ }^{2,4}$, A. FARAHAT ${ }^{1}$, AND A. VIGANÒ ${ }^{2,5}$
}

\begin{abstract}
We take into account two further physical models which play an utmost importance in the framework of Analogue Gravity. We first consider Bose-Einstein condensates (BEC) and then surface gravity waves in water. Our approach is based on the use of the master equation we introduced in a previous work. A more complete analysis of the singular perturbation problem involved, with particular reference to the behavior in the neighbourhood of the (real) turning point and its connection with the WKB approximation, allows us to verify the thermal character of the particle production process. Furthermore, we can provide a simple scheme apt to calculate explicitly the greybody factors in the case of BEC and surface waves. This corroborates the improved approach we proposed for studying the analogue Hawking effect in the usual limit of small dispersive effects.
\end{abstract}

\section{INTRODUCTION}

In 11 a possible unifying formalism was proposed for dealing with the analogous Hawking effect, based on a fourth-order equation of the Orr-Sommerfeld type extensively studied in a series of papers by Nishimoto (see $[2,3,4,5])$.

We take into consideration a further very interesting case, involving BEC and also surface waves, and provide both a sensible approximation scheme to the associated problems and also an analytical calculation of the greybody factor. We refer the reader to the following sections for an extensive list of references for theoretical studies of both the models. The utmost relevance of both the models is self-evident, due to the fact that BEC and water have been the most generally recognised benchmarks for experimental verification of the actual existence of Hawking radiation [6, 7, 8, 9, 10, 11, 12, 13. We do not discuss herein the problem represented by the subcritical case, which is left for future investigations.

In the following, we first take into account the Hawking effect in BEC. We adopt the healing length as the expansion parameter to be taken into account in order to deal with the problem of small dispersive effects. The well-known superluminal nature of the dispersion relation requires a different analysis with respect to the subluminal cases discussed in [1, with particular reference to the near horizon approximation. We obtain analytical solutions both in the far region and in the one near the turning point (horizon). We propose a different solution with respect to the ones existing in the literature, and obtain a complete analytical formula for inferring thermality and the grey-body factor.

In the second part of the paper we consider gravity waves in water. As expansion parameter we consider the shallowness parameter and, in this subluminal case, we again perform, by following analogous theoretical paths as above, the calculation of thermality and a scheme for the calculation of the grey-body factor. A further discussion follows.

\section{BEC}

There are several theoretical studies on analogous Hawking effect in BEC, starting from the seminal paper by Garay et al. [15. We limit ourselves to some relevant references concerning mainly semi-analytical/analytical approaches to the dispersive case [16, 17, 18, 19, 20, 21, 22, 23, 24, 25, 26].

We refer mainly to [17. For completeness, we reproduce some basic steps towards the equations we study in the following subsections. The field $\hat{\Psi}(t, \mathbf{x})$ of atoms of the condensate satisfies the commutation relations

$$
\left[\hat{\Psi}(t, \mathbf{x}), \hat{\Psi}^{\dagger}\left(t, \mathbf{x}^{\prime}\right)\right]=\delta^{3}\left(\mathbf{x}-\mathbf{x}^{\prime}\right)
$$

and the Heisenberg equation of motion

$$
[\hat{\Psi}(t, \mathbf{x}), \hat{H}]=i \hbar \partial_{t} \hat{\Psi}(t, \mathbf{x})
$$


where the Hamiltonian operator is

$$
\hat{H}=\int d^{3} x\left[\frac{\hbar^{2}}{2 m} \nabla_{\mathbf{x}} \hat{\Psi}^{\dagger} \nabla_{\mathbf{x}} \hat{\Psi}+V \hat{\Psi}^{\dagger} \hat{\Psi}+\frac{g}{2} \hat{\Psi}^{\dagger} \hat{\Psi}^{\dagger} \hat{\Psi} \hat{\Psi}\right]
$$

where $m$ is the mass of the atoms, $V$ is the external potential, and $g$ is an effective coupling [17. At very low temperatures, a condensed state forms, represented by a (c-number) state $\Psi_{0}$, and one may introduce also a relative (quantum) fluctuation in such a way that

$$
\hat{\Psi}=\Psi_{0}(1+\hat{\phi}),
$$

and then, considering only a 1-dimensional condensate henceforth, from the linearized equation one obtains

$$
i \hbar\left(\partial_{t}+v(x) \partial_{x}\right) \hat{\phi}=T \hat{\phi}+m c^{2}\left(\hat{\phi}+\hat{\phi}^{\dagger}\right),
$$

where $v(x), c(x)$ are the local flow velocity and the speed of sound, respectively, and for stationary condensates

$$
T:=-\hbar^{2} \frac{1}{2 m} v(x) \partial_{x}\left(\frac{1}{v(x)} \partial_{x}\right) .
$$

Focusing only on stationary condensates, one gets

$$
\hat{\phi}_{\omega}(t, x)=\hat{a}_{\omega} e^{-i \omega t} \phi_{\omega}(x)+\hat{a}_{\omega}^{\dagger}\left(e^{-i \omega t} \varphi_{\omega}(x)\right)^{*} .
$$

Then from 2.5 and the commutation relations for $\hat{a}_{\omega}, \hat{a}_{\omega}^{\dagger}$ one obtains for the stationary modes $\phi_{\omega}, \varphi_{\omega}$, which will be indicated as $\phi, \varphi$ henceforth, satisfy

$$
\begin{aligned}
\left(\hbar\left(\omega+i v(x) \partial_{x}\right)-T-m c^{2}(x)\right) \phi(x) & =m c^{2}(x) \varphi(x), \\
\left(-\hbar\left(\omega+i v(x) \partial_{x}\right)-T-m c^{2}(x)\right) \varphi(x) & =m c^{2}(x) \phi(x),
\end{aligned}
$$

It is straightforward to show that one may obtain separated equations for $\phi, \varphi$, as in [17]:

$$
\left[\left(\hbar\left(\omega+i v(x) \partial_{x}\right)+T\right) \frac{1}{c^{2}(x)}\left(-\hbar\left(\omega+i v(x) \partial_{x}\right)+T\right)+2 m T\right] \phi(x)=0,
$$

and

$$
\left[\left(-\hbar\left(\omega+i v(x) \partial_{x}\right)+T\right) \frac{1}{c^{2}(x)}\left(\hbar\left(\omega+i v(x) \partial_{x}\right)+T\right)+2 m T\right] \varphi(x)=0 .
$$

Both the above equations are fourth order ones, and henceforth we first take into account (2.10) for modes $\phi$. We notice that we can simplify a factor $\hbar^{2}$ overall. Furthermore, in order to eliminate the third order term, we put

$$
\phi(x)=c(x) \sqrt{v(x)} \zeta(x) .
$$

Then we obtain the equation

$$
\left[\alpha_{4}(x) \partial_{x}^{4}+\alpha_{2}(x) \partial_{x}^{2}+\alpha_{1}(x) \partial_{x}+\alpha_{0}(x)\right] \zeta(x)=0,
$$

where

$$
\begin{aligned}
\alpha_{4}(x)= & \frac{\hbar^{2}}{4 m^{2} c^{2}(x)}, \\
\alpha_{2}(x)= & -1+\frac{v^{2}(x)}{c^{2}(x)}+\ldots, \\
\alpha_{1}(x)= & \frac{2}{c^{2}(x)}\left(-i \omega v(x)-c(x) c^{\prime}(x)+v(x) v^{\prime}(x)\right)+\ldots \\
\alpha_{0}(x)= & \frac{1}{c^{2}(x)}\left(-\omega^{2}-2 \frac{v^{2}(x)\left(c^{\prime}(x)\right)^{2}}{c^{2}(x)}-i \omega v^{\prime}(x)+\frac{v(x) v^{\prime}(x) c^{\prime}(x)}{c(x)}+\frac{\left(v^{\prime}(x)\right)^{2}}{4}+\frac{3\left(v^{\prime}(x)\right)^{2} c^{2}(x)}{4 v^{2}(x)}\right. \\
& \left.-c^{\prime \prime}(x) c(x)+\frac{v^{2}(x) c^{\prime \prime}(x)}{c(x)}-\frac{v^{\prime \prime}(x) c^{2}(x)}{2 v(x)}+\frac{v(x) v^{\prime \prime}(x)}{2}\right)+\ldots
\end{aligned}
$$

In the above formulas, we did not write explicitly all the terms. The complete expression of the coefficients appears in appendix $\mathrm{A}$. 
A natural expansion parameter suitable for a weakly dispersive regime is the so-called healing length

$$
\xi:=\frac{\hbar}{\sqrt{2} m c(x)},
$$

which depends on the local speed of sound. Let us define

$$
\bar{\xi}=\sup _{x} \xi(x)=\frac{\hbar}{\sqrt{2} m} \frac{1}{\inf _{x} c(x)}=: \frac{\hbar}{\sqrt{2} m} \frac{1}{\bar{c}},
$$

where

$$
\bar{c}:=\inf _{x} c(x)>\epsilon>0 .
$$

Of course we have $0<\xi(x) \leq \bar{\xi}$, and $\bar{\xi} \rightarrow 0$ has to be meant as the limit of weak dispersive effects we are interested in 1 . We obtain the following fourth order equation of the Orr-Sommerfeld type $[2$

$$
\left[\bar{\xi}^{2} \partial_{x}^{4}-\left(\beta_{2}(x, \bar{\xi}) \partial_{x}^{2}+\beta_{1}(x, \bar{\xi}) \partial_{x}+\beta_{0}(x, \bar{\xi})\right)\right] \zeta(x)=0,
$$

where

$$
\begin{aligned}
\beta_{2}(x, \bar{\xi})= & \frac{2 c^{2}(x)}{\bar{c}^{2}}\left(1-\frac{v^{2}(x)}{c^{2}(x)}\right)+\left(-\frac{i \sqrt{2} \bar{c} v(x) c^{\prime}(x)}{c^{3}(x)}+\frac{i \sqrt{2} \bar{c} v^{\prime}(x)}{c^{2}(x)}\right) \bar{\xi}+O\left(\bar{\xi}^{2}\right), \\
\beta_{1}(x, \bar{\xi})= & \frac{4}{\bar{c}^{2}}\left(i \omega v(x)+c(x) c^{\prime}(x)-v(x) v^{\prime}(x)\right)+O(\bar{\xi}), \\
\beta_{0}(x, \bar{\xi})= & \frac{2}{\bar{c}^{2}}\left(\omega^{2}+2 \frac{v^{2}(x)\left(c^{\prime}(x)\right)^{2}}{c^{2}(x)}+i \omega v^{\prime}(x)-\frac{v(x) v^{\prime}(x) c^{\prime}(x)}{c(x)}-\frac{\left(v^{\prime}(x)\right)^{2}}{4}-\frac{3\left(v^{\prime}(x)\right)^{2} c^{2}(x)}{4 v^{2}(x)}\right. \\
& \left.+c^{\prime \prime}(x) c(x)-\frac{v^{2}(x) c^{\prime \prime}(x)}{c(x)}+\frac{v^{\prime \prime}(x) c^{2}(x)}{2 v(x)}-\frac{v(x) v^{\prime \prime}(x)}{2}\right)+O(\bar{\xi}) .
\end{aligned}
$$

2.1. The reduced equation. The reduced equation is

$$
\left(\beta_{2}(x, 0) \partial_{x}^{2}+\beta_{1}(x, 0) \partial_{x}+\beta_{0}(x, 0)\right) \zeta(x)=0,
$$

which displays a turning point (TP) such that

$$
\beta_{2}\left(x_{T P}, 0\right)=\left.0 \Longleftrightarrow\left(1-\frac{v^{2}(x)}{c^{2}(x)}\right)\right|_{x_{T P}}=0 .
$$

As in [17, we can assume $x_{T P}=0$ and get a black hole horizon for

$$
v(x)+c(x)=0,
$$

with $v<0$, and also in the linear region

$$
v(x)+c(x) \sim \kappa x,
$$

with $\kappa:=v^{\prime}(0)+c^{\prime}(0)>0$. As to 2.11), we point out that in the limit as $\bar{\xi} \rightarrow 0$, we obtain the same leading order contributions for $\varphi$ as for $\phi$. This is true for the results displayed in the following two subsections, so we shall not repeat the calculation also for $\varphi$.

2.2. WKB approximation. We put

$$
\zeta(x)=\exp \left(\frac{\theta(x)}{\bar{\xi}}\right) \sum_{n=0}^{\infty} \bar{\xi}^{n} y_{n}(x) .
$$

To the lowest order, we obtain

$$
{\theta^{\prime}}^{4} \bar{c}^{2}-2\left(c^{2}(x)-v^{2}(x)\right) \theta^{\prime 2}=0,
$$

whose solutions are $\theta^{\prime}=0$ (multiplicity two), and for $x<0$

$$
\theta_{ \pm}^{\prime}= \pm i \frac{\sqrt{2}}{\bar{c}} \sqrt{v^{2}(x)-c^{2}(x)} .
$$

\footnotetext{
${ }^{1}$ It should be more correctly intended as the limit where the healing length is much smaller than the wavelength of the perturbation on the background condensate 16
} 
As expected, due to the superluminal nature of the dispersion relation in BEC, two big wavenumber modes are found in the black hole region $x<0$, where $v^{2}(x)>c^{2}(x)$. We mention, in passing, that for $x>0$ the nonvanishing solutions correspond to the decaying mode and the growing mode respectively. As to the propagating solutions, we associate with them the so-called transport equation:

$$
\begin{aligned}
& \left(v^{2}(x)-c^{2}(x)+\bar{c}^{2}{\theta^{\prime}}^{2}(x)\right) y_{0}^{\prime}+\left(-i \omega v(x)-c(x) c^{\prime}(x)+v(x) v^{\prime}(x)\right. \\
& \left.+i \frac{\bar{c}}{\sqrt{2}}\left(v^{\prime}(x)-v(x) \frac{c^{\prime}(x)}{c(x)}\right) \theta^{\prime}(x)-\left(c^{2}(x)-v^{2}(x)\right) \frac{\theta^{\prime \prime}(x)}{2 \theta^{\prime}(x)}+\frac{3}{2} \bar{c}^{2} \theta^{\prime \prime}(x) \theta^{\prime}(x)\right) y_{0}(x)=0 .
\end{aligned}
$$

We then find the solutions

$$
y_{0 \pm}(x)=B_{ \pm}\left(v^{2}(x)-c^{2}(x)\right)^{-3 / 4}\left(\frac{v(x)}{c(x)}+\sqrt{\frac{v^{2}(x)}{c^{2}(x)}-1}\right)^{\mp 1} \exp \left(-i \omega \int^{x} d s \frac{v(s)}{v^{2}(s)-c^{2}(s)}\right) .
$$

In the near horizon region, it is easy to show that

$$
\left|y_{0 \pm}(x)\right| \propto x^{-3 / 4}
$$

as usual and expected. The high momentum modes are then

$$
\phi_{ \pm}(x)=c(x) \sqrt{v(x)} \zeta_{ \pm}(x)=c(x) \sqrt{v(x)} y_{0 \pm}(x) \exp \left(\frac{\theta_{ \pm}(x)}{\bar{\xi}}\right)
$$

and $\left|\phi_{ \pm}(x)\right| \propto x^{-3 / 4}$ near $x=0$, as in 2.29 . In particular, we have

$$
\zeta_{ \pm}^{w k b}(x) \sim-\left(2 c_{0} \kappa\right)^{-3 / 4}|x|^{-\frac{i \omega}{2 \kappa}-\frac{3}{4}} \exp \left(\mp \frac{2}{3} \frac{i}{\bar{\xi}} \sqrt{\frac{4 c_{0} \kappa}{\bar{c}^{2}}}|x|^{3 / 2}\right)
$$

where $c_{0}:=c(0)$; this formula will be useful in the following.

Two further solutions occurring when $\theta^{\prime}=0$ can be obtained from the reduced equation. First, we notice that near the turning point one obtains

$$
\left[\partial_{x}^{2}+\frac{1}{x}\left(1-i \frac{\omega}{\kappa}\right) \partial_{x}+(\ldots) \frac{1}{x}\right] \zeta(x)=0,
$$

where the coefficient $(. .$.$) does not contribute to the so-called indicial equation, whose roots are$

$$
\alpha_{1}=0, \quad \alpha_{2}=i \frac{\omega}{\kappa} \text {. }
$$

In particular, we can define [2]

$$
\lambda:=1-\alpha_{2}=1-i \frac{\omega}{\kappa} .
$$

We obtain near the regular singular point $x=0$ (our TP) the following series expansions: for $x>0$

$$
\begin{aligned}
& \phi_{v}(x)=1+\sum_{n=1}^{\infty} c_{n} x^{n}, \\
& \phi_{u}(x)=x^{i \frac{\omega}{\kappa}}\left(1+\sum_{n=1}^{\infty} d_{n} x^{n}\right) .
\end{aligned}
$$

The series expansion 2.35 holds true on both sides of the turning points, with different coefficients. So we can obtain also analogous expansions for the solutions $\phi_{d}(x)$ and $\phi_{l}(x)$ occurring for $x<0$. We omit the straightforward details. By comparing the behavior of the above four solutions in the so-called linear region where 2.23 holds, with the solutions one can obtain in the near turning point approximation (to be discussed in the following subsection), one finds the connection formulas providing the amplitudes for pair-creation we are interested in. See the following.

In particular, it is useful to provide also approximate solutions of the reduced equation as $x$ is large (in the external region with respect to the black hole). It is easy to show that for large $x$ in the above sense we have $v(x), c(x) \sim$ const., and then $v^{\prime}=0, c^{\prime}=0$. The asymptotic values of $v(x), c(x)$ as $x \rightarrow \infty$ are for simplicity 
indicated with $v_{r}, c_{r}$ respectively (analogously, one has $v \rightarrow v_{l}, c \rightarrow c_{l}$ for $x \rightarrow-\infty$ ). As a consequence e.g. under the conditions of theorem 1.9.1 of [27, we get asymptotically for $x \rightarrow \infty$

$$
\begin{aligned}
& \phi_{v}(x) \sim \exp \left(-i \frac{\omega}{c_{r}-v_{r}} x\right), \\
& \phi_{u}(x) \sim \exp \left(i \frac{\omega}{c_{r}+v_{r}} x\right) .
\end{aligned}
$$

Analogously, for $x \rightarrow-\infty$ one obtains

$$
\begin{aligned}
& \phi_{d}(x) \sim \exp \left(-i \frac{\omega}{c_{l}-v_{l}} x\right), \\
& \phi_{l}(x) \sim \exp \left(i \frac{\omega}{c_{l}+v_{l}} x\right) .
\end{aligned}
$$

We note that $\phi_{l}(x)$ is a negative-norm mode.

2.3. Approximation near the turning point. Solutions near the TP have to satisfy the following equation, as shown in [1]:

$$
\frac{d^{4} \zeta}{d z^{4}}-\left(z \frac{d^{2} \zeta}{d z^{2}}+\lambda \frac{d \zeta}{d z}\right)=0
$$

where

$$
\lambda:=1-i \frac{\omega}{\kappa},
$$

and

$$
z=\left(\frac{4 c_{0}}{\bar{c}^{2}} \kappa\right)^{1 / 3} \epsilon^{-2 / 3} x
$$

where $c_{0}:=c(0)$. There is a first solution which is constant, and put equal to one (cf. 2]). This solution represents the near horizon approximation for the counter-propagating mode $v$ discussed in the previous subsection and, albeit nearly trivial, it is fundamental for getting a complete basis for solutions near the turning point. Further solutions of equation 2.41 can be found by means of Laplace integrals

$$
\zeta_{j}(z)=\frac{1}{2 \pi i} \int_{C_{j}} d t t^{\lambda-2} \exp \left(z t-\frac{1}{3} t^{3}\right),
$$

with a suitable choice for the paths $C_{j}$ in the complex $t$-plane. For the superluminal case at hand the aforementioned solutions of (2.44) are also known as generalized Airy functions.

Paths extending to infinity in the complex $t$-plane must be restricted to allowed regions. We have the same regions as for the Airy functions, with $\theta:=\arg (t)$ :

$$
\theta \in\left(-\frac{\pi}{6}, \frac{\pi}{6}\right) \cup\left(\frac{\pi}{2}, \frac{5 \pi}{6}\right) \cup\left(\frac{7 \pi}{6}, \frac{3 \pi}{2}\right) .
$$

The boundary conditions we introduce herein differ from the ones of the seminal investigation for a superluminal model contained in [14, and also from the ones in [28. Some differences appear also with the analysis of [29, 30] for the Corley model, where the same diagram was proposed, and where, furthermore Fourier transform was used in place of Laplace transform.

Our choice is the following: in order to describe the states in the external region $x>0$, which correspond to the decaying mode and to the cut-mode related to the Hawking particle, we choose to introduce the cut in the positive real axis in the complex $t$-plane.

One may consider as boundary condition the presence of the Hawking mode (cut mode) and of the decaying mode (albeit it does not participate to fluxes at infinity, it may play a role for local observables [29]). The corresponding paths are homotopic to the ones for the two states inside, which correspond to the big wavenumber $k_{ \pm}$states in the black hole region travelling towards the horizon. See also figure1. We then obtain the 'Corley's diagram' for the creation process of Hawking particles. In our interpretation, at the level of the particle creation process, the fourth mode $v$ is not directly involved. Still, it plays a role in depleting the Hawking particle flux only in a further process of scattering on the geometry associated with the reduced equation, in analogy to our 
discussion for the cases of the subluminal Corley model and of the dielectric model taken into account in [1]. This is in agreement with the analysis in 29, 30] for the Corley model. Cf. [1], figure 2 therein.

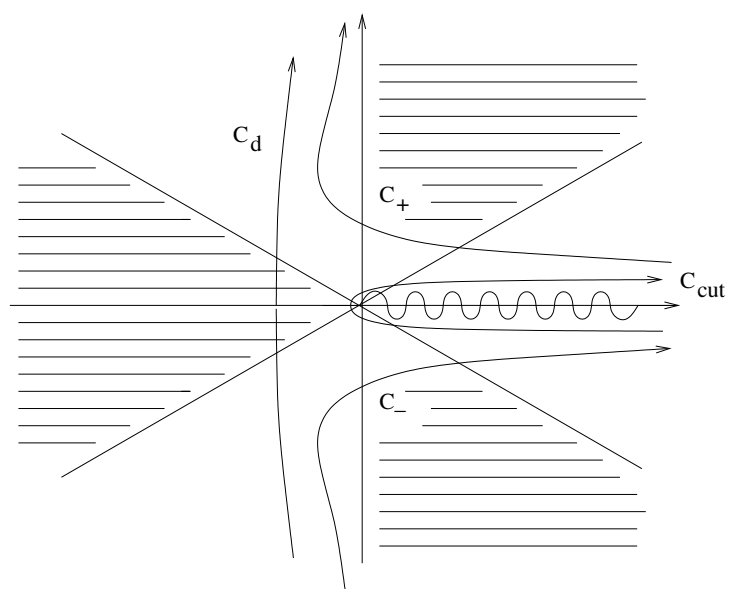

Figure 1. Paths for the superluminal case. $C_{d}, C_{c u t}$ are the decaying mode and the Hawking mode respectively, and are found in the external region $x>0 . C_{ \pm}$correspond to the dispersive modes, and are found in the black hole region $x<0 . C_{d}, C_{c u t}$ can be deformed into $C_{+}, C_{-}$.

It is important to note that $\zeta_{j}(z)$ can be rewritten as follows: by putting $t=\sqrt{|z|} u$, we get

$$
\zeta_{j}(z)=\frac{1}{2 \pi i}|z|^{\frac{\lambda-1}{2}} I_{j}(z)
$$

where

$$
I_{j}(z)=\int_{\bar{C}_{j}} d u g(u) \exp \left(|z|^{3 / 2} h_{ \pm}(u)\right)
$$

and

$$
\begin{aligned}
g(u) & :=u^{\lambda-2}, \\
h_{ \pm}(u) & := \pm u-\frac{u^{3}}{3} .
\end{aligned}
$$

For the decaying mode, which passes through the saddle point at $u=1$ (in the external region $h_{+}$is involved) we get

$$
\zeta_{\text {decaying }}(z) \simeq e^{-i \pi} e^{\frac{\pi \omega}{\kappa}} \frac{1}{2 \sqrt{\pi}}|z|^{-\frac{i \omega}{2 \kappa}-\frac{3}{4}} e^{-\frac{2}{3}|z|^{3 / 2}} .
$$

As to the cut mode, we stress that the branch cut lies along a steepest descent. Indeed, we have that the imaginary part of $u-u^{3} / 3$ is $b\left(1-a^{2}+b^{2} / 3\right)$, where $a, b$ are the real part and the imaginary part of $u$ respectively. As a consequence, $b=0$ is a steepest descent line. This allows us to calculate the cut contribution along the lines suggested in 31, chapter 4 , section 4.8, finding thus

$$
\zeta_{c u t}(z) \simeq-\frac{1}{i \pi}|z|^{i \frac{\omega}{k}} \Gamma\left(-i \frac{\omega}{\kappa}\right) \sinh \left(\frac{\pi \omega}{\kappa}\right) .
$$

For $x<0$ we have the modes $k_{ \pm}$in correspondence of the steepest descents passing through the saddle points $u_{ \pm}= \pm i$. Then we get (cf. also [14, 29, 30] for the Corley model), with a coincidence that is related to the universal character of our near-horizon equation, which holds true also for the Corley model [1]

$$
\begin{aligned}
& \zeta_{-}(z) \simeq \frac{1}{2 \sqrt{\pi}} e^{\frac{1}{4} \pi i} e^{-\frac{\pi \omega}{2 \kappa}}|z|^{-\frac{i \omega}{2 \kappa}-\frac{3}{4}} e^{i \frac{2}{3}|z|^{3 / 2}}, \\
& \zeta_{+}(z) \simeq-\frac{1}{2 \sqrt{\pi}} e^{\frac{3}{4} \pi i} e^{\frac{\pi \omega}{2 \kappa}}|z|^{-\frac{i \omega}{2 \kappa}-\frac{3}{4}} e^{-i \frac{2}{3}|z|^{3 / 2}} .
\end{aligned}
$$


The black hole boundary condition near the horizon forces the coefficients of the modes \pm and $u$ to be equal; by appealing to the Cauchy theorem, and to the fact that all the functions in the near-horizon approximation are well-defined for $z=0$, we have

$$
\zeta_{+}(0)+\zeta_{-}(0)+\zeta_{u}(0)=\zeta_{\text {decaying }}(0) .
$$

As discussed in [1, this condition amounts to the Corley's ansatz, i.e. the black hole boundary condition. The fourth mode does not appear in the diagram, and its contribution is zero at the level of the pair-creation process. See [1] for a further discussion. See also below. Then, by comparing with the WKB solutions in the matching region, we find for the propagating part of the field

$$
\begin{aligned}
\phi(x, t)= & \left(e^{\frac{3}{4} \pi i} \frac{e^{\frac{\pi \omega}{2 \kappa}}}{2 \sqrt{\pi}} 2^{1 / 4} \sqrt{c_{0} \kappa \bar{c}}\left(\frac{4 c_{0} \kappa}{\bar{c}^{2}}\right)^{-\frac{i \omega}{6 \kappa}} \bar{\xi}^{\frac{i \omega}{3 \kappa}+\frac{1}{2}} \phi_{+}(x, t)\right. \\
& \left.+e^{\frac{1}{4} \pi i} \frac{e^{-\frac{\pi \omega}{2 \kappa}}}{2 \sqrt{\pi}} 2^{1 / 4} \sqrt{c_{0} \kappa \bar{c}}\left(\frac{4 c_{0} \kappa}{\bar{c}^{2}}\right)^{-\frac{i \omega}{6 \kappa}} \bar{\xi}^{\frac{i \omega}{3 \kappa}+\frac{1}{2}} \phi_{-}(x, t)\right) \theta(-x) \\
& +\left(-\frac{\sinh \left(\frac{\pi \omega}{\kappa}\right)}{\pi i} \Gamma\left(-i \frac{\omega}{\kappa}\right)\left(\frac{4 c_{0} \kappa}{\bar{c}^{2}}\right)^{\frac{i \omega}{3 \kappa}} \bar{\xi}^{-\frac{2 i \omega}{3 \kappa}} \phi_{u}(x, t)+h \phi_{v}(x, t)\right) \theta(x),
\end{aligned}
$$

where $\phi_{ \pm}, \phi_{u}, \phi_{v}$ are the WKB solutions for the modes at hand. As to the modes $d, l$, one may proceed as in the Corley model discussed in [1]. We do not delve into the details.

2.4. Calculations for the $\varphi$ stationary modes. In place of (2.10) one must consider (2.11), and again the parameter for asymptotic expansion is $\bar{\xi}$. We do not repeat all the steps, and we limit ourselves to point out some features. In order to eliminate the third order term, one puts again

$$
\varphi(x)=c(x) \sqrt{v(x)} \eta(x) .
$$

As to the WKB approximation, we note that equation for $\theta$ remains the same as for $\phi$, and that for the long wavenumber modes one obtains again (2.26). For the transport equation only a change occurs,

$$
\begin{aligned}
& \left(v^{2}(x)-c^{2}(x)+\bar{c}^{2} \theta^{\prime 2}(x)\right) y_{0}^{\prime}+\left(-i \omega v(x)-c(x) c^{\prime}(x)+v(x) v^{\prime}(x)\right. \\
& \left.-i \frac{\bar{c}}{\sqrt{2}}\left(v^{\prime}(x)-v(x) \frac{c^{\prime}(x)}{c(x)}\right) \theta^{\prime}(x)-\left(c^{2}(x)-v^{2}(x)\right) \frac{\theta^{\prime \prime}(x)}{2 \theta^{\prime}(x)}+\frac{3}{2} \bar{c}^{2} \theta^{\prime \prime}(x) \theta^{\prime}(x)\right) y_{0}(x)=0 .
\end{aligned}
$$

We then find the solutions

$$
y_{0 \pm}(x)=B\left(v^{2}(x)-c^{2}(x)\right)^{-3 / 4}\left(\frac{v(x)}{c(x)}+\sqrt{\frac{v^{2}(x)}{c^{2}(x)}-1}\right)^{ \pm 1} \exp \left(-i \omega \int^{x} d s \frac{v(s)}{v^{2}(s)-c^{2}(s)}\right) .
$$

This does not substantially modify the expansion in the linear region. As to the reduced equation, one has again

$$
\left[\partial_{x}^{2}+\frac{1}{x}\left(1-i \frac{\omega}{\kappa}\right) \partial_{x}+\frac{1}{x}(\ldots)\right] \eta(x)=0,
$$

whose indicial equation is the same as in the previous sections, and also the solutions remain the same in the asymptotic region.

Solutions near the TP have to satisfy the same equation as for the other mode 2.41), simply through the substitution $\zeta \mapsto \eta$. As a consequence, also the near horizon solutions remain the same, and also the matching formulas in the linear region do not change.

2.5. Thermality. We recall that with the stationary modes $\phi, \varphi$ one can associate conserved currents (see [32] and 18 for an application to the analogous Hawking radiation):

$$
J_{x}^{\left[\phi_{a}, \varphi_{a}\right]}:=-i \frac{\hbar}{2 m}\left(\phi_{a}^{*} \partial_{x} \phi_{a}-\phi_{a} \partial_{x} \phi_{a}^{*}+\varphi_{a}^{*} \partial_{x} \varphi_{a}-\varphi_{a} \partial_{x} \varphi_{a}^{*}\right),
$$


where we have $a= \pm, u, v$. In the following, we indicate simply with $J_{x}$ the above currents, and we mean to exploit the following current flux conservation:

$$
\left|J_{x}^{u}\right|=\left|J_{x}^{+}\right|-\left|J_{x}^{-}\right|+\left|J_{x}^{v}\right|,
$$

where the outgoing flux of Hawking particles ( $u$-modes, directed towards $\infty$ ) originates from the ingoing flux of modes ( $v$ and $k_{ \pm}$modes, directed towards the horizon $x=0$ ), and the nature of the modes $k_{-}$to be negative norm modes has be taken into account.

The normalization to the modes is as in [17, 22, by requiring that in the eikonal approximation the dispersion relation (which holds with constant coefficients in the asymptotic regions)

$$
(\omega-v k)^{2}=c^{2} k^{2}\left(1+\frac{\xi^{2}}{2} k^{2}\right),
$$

holds true. The WKB solutions, as $|x| \rightarrow \infty$, behave as plane waves which we indicate as $\phi_{\omega}, \varphi_{\omega}$ for simplicity (omitting for the moment any further mode label). We take into account that the two components $\left(\phi_{\omega}, \varphi_{\omega}\right)$ satisfy the equations of motion 2.8 and 2.9 , and then we get 22

$$
\begin{aligned}
& \phi_{\omega}=D_{\omega} e^{-i \omega t+i k(\omega) x}=N_{\omega}\left(\omega-v k+c \frac{\xi}{\sqrt{2}} k^{2}\right) e^{-i \omega t+i k(\omega) x}, \\
& \varphi_{\omega}=E_{\omega} e^{-i \omega t+i k(\omega) x}=N_{\omega}\left(-\left(\omega-v k-c \frac{\xi}{\sqrt{2}} k^{2}\right)\right) e^{-i \omega t+i k(\omega) x},
\end{aligned}
$$

where

$$
N_{\omega}=\frac{1}{\sqrt{4 \sqrt{2} \pi \hbar \rho c \xi k^{2}\left|(\omega-v k)\left(\frac{d k(\omega)}{d \omega}\right)^{-1}\right|}},
$$

with $\rho \propto 1 / v$. These normalization factors in the asymptotic region reduce to the ones of the homogeneous BEC, of course.

For explicit calculations, we point out that for each mode it holds

$$
\left|J_{x}\right| \propto k\left(\left|D_{\omega}\right|^{2}+\left|E_{\omega}\right|^{2}\right) \propto(\omega-v k)^{2}+\left(c \frac{\xi}{\sqrt{2}} k^{2}\right)^{2} .
$$

As usual, for thermality $\frac{\left|J_{x}^{-}\right|}{\left|J_{x}^{+}\right|}=e^{-\beta \omega}$ holds, where $\beta=\frac{2 \pi}{\kappa}$ is the inverse Hawking temperature.

2.6. Grey-body factor. As to the grey-body factor, in [1] it has also been shown that, in principle, one might deduce the grey-body factor from the direct calculation of

$$
\left|\beta_{\omega}\right|^{2}:=\frac{\left|J_{x}^{-}\right|}{\left|J_{x}^{u}\right|},
$$

which represents the number of created particles, as well-known. Even if this route is viable, the drawback is that there is the risk of a poor approximation (as in the standard Hawking effect calculations).

The grey-body factor can be obtained as follows:

$$
\Gamma=1-R,
$$

where we also define the ratio

$$
R:=\frac{\left|J_{x}^{v}\right|}{\left|J_{x}^{u}\right|}
$$

As discussed in [1], actually the mode $v$ does not participate directly to the Hawking pair-creation process. Still, there can be a further contribution to $R$ arising from the back-scattering on the geometry, leaving room for $\Gamma<1$. Then, $R$ represents the reflection coefficient for the scattering of Hawking particles in the background geometry associated with the reduced equation obtained for $\bar{\xi}=0$. This is in agreement with what happens in the Corley 
model [29]. Given a $u$-mode entering from the part of the linear region, where the WKB approximation is valid, the reduced equation provides the contribution

$$
R_{\text {reduced }}:=\left(\frac{\left|J_{x}^{v}\right|}{\left|J_{x}^{u}\right|}\right)_{\text {reduced }}
$$

with the fluxes computed asymptotically, and with $\left|J_{x}^{v}\right|$ measured near the horizon, but still in a region where the WKB works well). See [1] for a complete discussion. As the aforementioned geometry amounts to the classical geometry for BEC analogous black holes, we refer to the expressions already present in the literature and calculated in the so-called hydrodynamic limit, see in particular 25]. Of course, also in this case there exists a maximal frequency $\omega_{\max }$ such that, for $\omega>\omega_{\max }$, only two modes participates to the scattering process and the Hawking effect is no more present [17, so that the spectrum is truncated at $\omega_{\max }$ for non-zero values of $\bar{\xi}$.

\section{SHALlow WATER WAVES}

Shallow water waves are the other fundamental benchmark of analogue gravity as, just for the case of BEC, experimental measurements of the analogous Hawking effect were carried out 7, 9, 10. Theoretical studies start with [33] and have been deepened further on. Also the phenomenon of undulation has been studied in detail [34, as well as the problem of the subcritical case 35, 36, 37, 38. We discuss herein only the transcritical case, which is the one properly associated with the analogous Hawking effect (although the subcritical case may preserve some imprinting of the Hawking phenomenon [37). Furthermore, we refer to the model discussed in [37.

As in [37] (cf. also 34]), we limit ourselves to the weakly dispersive case where

$$
\tanh \left(i h \partial_{x}\right) \mapsto i h \partial_{x}+i \frac{1}{3} h^{3} \partial_{x}^{3}
$$

$h$ is the local height (depth) of water. The corresponding (approximate) action is [37]

$$
S=\frac{1}{2} \int d^{2} x\left[\left(\left(\partial_{t}+v(x) \partial_{x}\right) \phi\right)^{2}-c^{2}(x)\left(\partial_{x} \phi\right)^{2}+\frac{g h^{3}(x)}{3}\left(\partial_{x}^{2} \phi\right)^{2}\right],
$$

where $v(x)$ is the local velocity of the fluid, $c(x)$ is the local speed of sound, $h(x)$ is the local height and $g$ is the gravity acceleration. This is the case most similar to the original model studied in [14, 29, and is characterized by a subluminal dispersion, as well known. The equation of motion for stationary modes $\phi(t, x)=e^{-i \omega t} \psi(x)$ is a quartic equation of the following form:

$$
\left(\frac{g}{3} h^{3} \partial_{x}^{4}+2 g h^{2} h^{\prime} \partial_{x}^{3}+\left[\left(c^{2}-v^{2}\right)+g\left(2 h\left(h^{\prime}\right)^{2}+h^{2} h^{\prime \prime}\right)\right] \partial_{x}^{2}+2\left(i \omega v+c c^{\prime}-v v^{\prime}\right) \partial_{x}+\left(\omega^{2}+i v^{\prime} \omega\right)\right) \psi=0 .
$$

It is also to be noted that the model is associated with a peculiar conserved current for stationary modes

$$
J_{x}=\operatorname{Im}\left[i \omega v \psi^{*} \psi+\left(c^{2}-v^{2}\right) \psi^{*} \partial_{x} \psi+\frac{g}{3} \psi^{*} \partial_{x}\left(h^{3} \partial_{x}^{2} \psi\right)-\frac{g}{3} h^{3}\left(\partial_{x} \psi^{*}\right)\left(\partial_{x}^{2} \psi\right)\right],
$$

as in [37, 39].

3.1. Rescaled variables. It is useful to proceed as in [40, by defining rescaled adimensional variables $z, \tau$ in place of $x, t$ as follows:

$$
\begin{aligned}
& x=\lambda s, \\
& t=\frac{\lambda}{\sqrt{g h_{0}}} \tau,
\end{aligned}
$$


where $\lambda$ stays for the wavelength and $h_{0}$ is to be considered a reference height (we could assume, for example, $h_{0}=\inf _{x} h(x)>0$. See also below). We can also introduce

$$
\begin{aligned}
v(x) & =\sqrt{g h_{0}} \bar{v}(x), \\
c(x) & =\sqrt{g h_{0}} \bar{c}(x), \\
\omega & =\frac{\sqrt{g h_{0}}}{\lambda} \bar{\omega},
\end{aligned}
$$

as well as the so-called long wavelength or shallowness parameter [40]

$$
\delta:=\frac{h_{0}}{\lambda} .
$$

As a consequence, from equation $(3.3)$, we obtain

$$
\begin{aligned}
& \left(\delta^{2}\left(\partial_{s}^{4}+6 \frac{h^{\prime}}{h} \partial_{s}^{3}\right)+\right. \\
& \left.\left[3 \frac{h_{0}^{3}}{h^{3}}\left(\bar{c}^{2}-\bar{v}^{2}\right)+\delta^{2}\left(6 \frac{\left(h^{\prime}\right)^{2}}{h^{2}}+3 \frac{h^{\prime \prime}}{h}\right)\right] \partial_{s}^{2}+6 \frac{h_{0}^{3}}{h^{3}}\left(i \bar{v} \bar{\omega}+\bar{c} \bar{c}^{\prime}-\bar{v} \bar{v}^{\prime}\right) \partial_{s}+3 \frac{h_{0}^{3}}{h^{3}}\left(\bar{\omega}^{2}+i \bar{v}^{\prime} \bar{\omega}\right)\right) \psi=0,
\end{aligned}
$$

where, with some liberal attitude, a prime indicates the derivative with respect to the dimensionless variable $s$. The third-order term can be removed by means of the following Liouville-like transformation

$$
\psi=h^{-3 / 2} \zeta
$$

which allows to obtain

$$
\left[\delta^{2} \partial_{s}^{4}+\gamma_{2}(s, \delta) \partial_{s}^{2}+\gamma_{1}(s, \delta) \partial_{s}+\gamma_{0}(s, \delta)\right] \zeta(s)=0
$$

where

$$
\begin{aligned}
\gamma_{2}(s, \delta)= & 3 \frac{h_{0}^{3}}{h^{3}(s)}\left(\bar{c}^{2}(s)-\bar{v}^{2}(s)\right)+O\left(\delta^{2}\right), \\
\gamma_{1}(s, \delta)= & 6 \frac{h_{0}^{3}}{h^{3}(s)}\left(i \bar{v}(s) \bar{\omega}+\bar{c}(s) \bar{c}^{\prime}(s)-\bar{v}(s) \bar{v}^{\prime}(s)\right)-9 \frac{h_{0}^{3} h^{\prime}(s)}{h^{4}(s)}\left(\bar{c}^{2}(s)-\bar{v}^{2}(s)\right)+O\left(\delta^{2}\right), \\
\gamma_{0}(s, \delta)= & 3 \frac{h_{0}^{3}}{h^{3}(s)}\left(\bar{\omega}^{2}+i \bar{v}^{\prime}(s) \bar{\omega}\right)+9 \frac{h_{0}^{3}}{h^{4}(s)}\left[-i \bar{\omega} \bar{v}(s) h^{\prime}(s)-\left(\bar{c}(s) \bar{c}^{\prime}(s)-\bar{v}(s) \bar{v}^{\prime}(s)\right) h^{\prime}(s)\right. \\
& \left.-\frac{1}{2}\left(\bar{c}^{2}(s)-\bar{v}^{2}(s)\right)\left(h^{\prime \prime}(s)+\frac{5}{2} \frac{\left(h^{\prime}(s)\right)^{2}}{h^{2}(s)}\right)\right]+O\left(\delta^{2}\right) .
\end{aligned}
$$

3.2. The reduced equation. The reduced equation is

$$
\left(\gamma_{2}(s, 0) \partial_{s}^{2}+\gamma_{1}(s, 0) \partial_{s}+\gamma_{0}(s, 0)\right) \zeta(s)=0,
$$

which displays a turning point (TP) such that

$$
\gamma_{2}\left(s_{T P}, 0\right)=\left.0 \Longleftrightarrow\left(\bar{v}^{2}(s)-\bar{c}^{2}(s)\right)\right|_{s_{T P}}=0 .
$$

As usual, by returning momentarily to dimensionful variables, we can assume $x_{T P}=0$ and get a black hole horizon for $v(x)+c(x)=0$, nwith $v<0$, and also in the linear region $v(x)+c(x) \sim \kappa x$, with $\kappa=v^{\prime}(0)+c^{\prime}(0)$. Also in this case near the TP one obtains

$$
\left[\partial_{x}^{2}+\frac{1}{x}\left(1-i \frac{\omega}{\kappa}\right) \partial_{x}+\frac{1}{x}(\ldots)\right] \zeta(x)=0
$$

where the coefficient $(\ldots)$ does not contribute to the so-called indicial equation, whose roots are again

$$
\alpha_{1}=0, \quad \alpha_{2}=i \frac{\omega}{\kappa} .
$$

and, again

$$
\mu:=1-\alpha_{2}=1-i \frac{\omega}{\kappa} .
$$


3.3. WKB approximation. We put

$$
\zeta(s)=\exp \left(\frac{\theta(s)}{\delta}\right) \sum_{n=0}^{\infty} \delta^{n} y_{n}(s)
$$

To the lowest order, we obtain

$$
\theta^{\prime 4}+3\left(\frac{h_{0}}{h}\right)^{3}\left(\bar{c}^{2}-\bar{v}^{2}\right) \theta^{\prime 2}=0
$$

whose solutions are $\theta^{\prime}=0$ (multiplicity two), and

$$
\theta_{ \pm}^{\prime}= \pm i \sqrt{3\left(\frac{h_{0}}{h}\right)^{3}} \sqrt{\bar{c}^{2}-\bar{v}^{2}}
$$

We first take into account the latter solutions, and associate with them the so-called transport equation

$$
\left(6\left(\frac{h_{0}}{h}\right)^{3}\left(\bar{c}^{2}-\bar{v}^{2}\right)+4{\theta^{\prime}}^{2}\right) y_{0}^{\prime}+\left(6 \theta^{\prime} \theta^{\prime \prime}+3\left(\frac{h_{0}}{h}\right)^{3}\left(2 i \bar{v} \bar{\omega}+2\left(\bar{c} \bar{c}^{\prime}-\bar{v} \bar{v}^{\prime}\right)+\left(\frac{\theta^{\prime \prime}}{\theta^{\prime}}-3 \frac{h^{\prime}}{h}\right)\left(\bar{c}^{2}-\bar{v}^{2}\right)\right)\right) y_{0}=0
$$

We then find the solutions

$$
y_{0 \pm}(s)=B\left(\bar{c}^{2}-\bar{v}^{2}\right)^{-3 / 4} h^{9 / 4} \exp \left(i \bar{\omega} \int^{s} \frac{\bar{v}}{\bar{c}^{2}-\bar{v}^{2}}\right) .
$$

As to the degenerate solutions with $\theta^{\prime}=0$, as known, they must solve the reduced equation with $\delta=0$ (3.15). We obtain near the regular singular point $s=0$ (our TP) the series expansions for $s>0$

$$
\begin{aligned}
& \psi_{v}(s)=1+\sum_{n=1}^{\infty} c_{n} s^{n}, \\
& \psi_{u}(s)=s^{i \frac{\omega}{\kappa}}\left(1+\sum_{n=1}^{\infty} d_{n} s^{n}\right) .
\end{aligned}
$$

In particular, it is useful to provide also approximate solutions of the reduced equation as $s$ is large (in the external region with respect to the black hole). It is easy to show that for large $s \rightarrow \infty$ in the above sense, we have $v(x), c(x) \sim$ const., and then $v^{\prime}=0, c^{\prime}=0$. The asymptotic values of $\bar{v}(x), \bar{c}(x)$ as $x \rightarrow \infty$ are for

simplicity indicated with $\bar{v}, \bar{c}$ respectively. As a consequence e.g. under the conditions of theorem 1.9.1 of [27], we get asymptotically for $s \rightarrow \infty$

$$
\begin{aligned}
& \psi_{v}(s) \sim \exp \left(-i \frac{\bar{\omega}}{\bar{c}-\bar{v}} s\right), \\
& \psi_{u}(s) \sim \exp \left(i \frac{\bar{\omega}}{\bar{c}+\bar{v}} s\right) .
\end{aligned}
$$

As in the previous model, we can also study solutions for $s<0$, and the only propagating ones asymptotically behave as

$$
\begin{aligned}
& \psi_{d}(s) \sim \exp \left(-i \frac{\bar{\omega}}{\bar{c}_{l}-\bar{v}_{l}} s\right), \\
& \psi_{l}(s) \sim \exp \left(i \frac{\bar{\omega}}{\bar{c}_{l}+\bar{v}_{l}} s\right)
\end{aligned}
$$

where $\bar{v}_{l}, \bar{c}_{l}$ are the limits of $\bar{v}(s), \bar{c}(s)$ for $x \rightarrow-\infty$ respectively. 
3.4. Near-horizon approximation. Near the TP the following equation holds true (cf. [1]):

$$
\frac{d^{4} \zeta}{d z^{4}}+\left(z \frac{d^{2} \zeta}{d z^{2}}+\mu \frac{d \zeta}{d z}\right)=0
$$

where $\mu$ is given in 3.19 , and

$$
z=\frac{h_{0}}{h(0)}\left(6 \bar{\kappa} \bar{c}_{0}\right)^{1 / 3} \delta^{-2 / 3} s
$$

where $\bar{\kappa}:=\kappa \sqrt{g h_{0}} / \lambda$ and $\bar{c}_{0}:=\bar{c}(0)$. We may also choose $h_{0}=h(0)$, i.e. we can assume that $h_{0}$ is the value of $h$ at the TP.

Apart for the constant solution, which is again put equal to one (cf. 2]), further solutions of equation (3.31) can be found by means of Laplace integrals as in [14, 28,

$$
\zeta_{j}(z)=\frac{1}{2 \pi i} \int_{C_{j}} d t t^{\mu-2} \exp \left(z t+\frac{1}{3} t^{3}\right)
$$

with a suitable choice for the paths $C_{j}$ in the complex $t$-plane. In this case, a subluminal character of the nonlinear part is present. Paths extending to infinity in the complex $t$-plane must be restricted to allowed regions, displayed in [14, 1] and, with $\theta:=\arg (t)$, we obtain:

$$
\theta \in\left(\frac{\pi}{6}, \frac{\pi}{2}\right) \cup\left(\frac{5 \pi}{6}, \frac{7 \pi}{6}\right) \cup\left(\frac{3 \pi}{2}, \frac{11 \pi}{6}\right) .
$$

We start from (3.33) and follow the general method explained in 11. Paths can be chosen as in any subluminal case, cf. figure 2 in [1. It corresponds to the so-called black hole boundary condition as discussed in 14. See also [29, 30]. We limit ourselves to reproduce the results. We have for $x<0$ the decaying mode

$$
w_{\text {decaying }}(z) \simeq \frac{1}{2 \sqrt{\pi}}|z|^{-\frac{i \omega}{2 \kappa}-\frac{3}{4}} e^{-\frac{2}{3}|z|^{3 / 2}} .
$$

As usual [14, it provides the aforementioned black hole boundary condition. For $x>0$ we have the modes $k_{ \pm}$ in correspondence of the steepest descents

$$
\begin{aligned}
& w_{+}(z) \simeq \frac{1}{2 \sqrt{\pi}} e^{-\frac{3}{4} \pi i} e^{\frac{\pi \omega}{2 \kappa}}|z|^{-\frac{i \omega}{2 \kappa}-\frac{3}{4}} e^{i \frac{2}{3}|z|^{3 / 2}}, \\
& w_{-}(z) \simeq \frac{1}{2 \sqrt{\pi}} e^{\frac{1}{4} \pi i} e^{-\frac{\pi \omega}{2 \kappa}}|z|^{-\frac{i \omega}{2 \kappa}-\frac{3}{4}} e^{-i \frac{2}{3}|z|^{3 / 2}} .
\end{aligned}
$$

The cut contribution, on the negative real axis, represents the Hawking mode and can be also in this case calculated along the lines suggested in [31], chapter 4, section 4.8:

$$
w_{\text {cut }}(z) \simeq-\frac{1}{i \pi}|z|^{i \frac{\omega}{\kappa}} \Gamma\left(-i \frac{\omega}{\kappa}\right) \sinh \left(\frac{\pi \omega}{\kappa}\right) .
$$

We are interested in connecting the expansions of the near horizon approximation with the ones of the WKB approximation displayed in the previous section. We get

$$
\psi(s, \tau)=c_{+} \psi_{+}(s, \tau)+c_{-} \psi_{-}(s, \tau)+c_{u} \phi_{u}(s, \tau)+c_{v} \phi_{v}(s, \tau),
$$

where the fourth mode gives no direct contribution to the pair-creation associated with the Hawking effect. Cf. 1]. We also have

$$
\psi_{j}(s, \tau)=\exp \left(-i \bar{\omega} \tau+i \bar{k}_{j}(\omega) \tau\right)
$$

with $j= \pm u, v$ and $\bar{k}_{j}(\omega)$ is the corresponding rescaled wavenumber. By comparing with the WKB solutions again in the matching region

$$
\begin{aligned}
\psi(s, \tau)= & e^{-\frac{3}{4} \pi i}(h(0))^{-9 / 4} 3^{-1 / 4} \sqrt{2 \overline{c_{0}} \bar{\kappa}} \frac{e^{\frac{\pi \omega}{\kappa}}}{2 \sqrt{\pi}}\left(6 \overline{c_{0}} \bar{\kappa}\right)^{-\frac{i \omega}{6 \kappa}} \delta^{\frac{i \omega}{3 \kappa}+\frac{1}{2}} \psi_{+}(s, \tau) \\
& +e^{\frac{1}{4} \pi i}(h(0))^{-9 / 4} 3^{-1 / 4} \sqrt{2 \overline{c_{0}} \bar{\kappa}} \frac{e^{-\frac{\pi \omega}{\kappa}}}{2 \sqrt{\pi}}\left(6 \overline{c_{0}} \bar{\kappa}\right)^{-\frac{i \omega}{6 \kappa}} \delta^{\frac{i \omega}{3 \kappa}+\frac{1}{2}} \psi_{-}(s, \tau) \\
& -\frac{\sinh \left(\frac{\pi \omega}{\kappa}\right)}{\pi i} \Gamma\left(-\frac{i \omega}{\kappa}\right)\left(6 \overline{c_{0}} \bar{\kappa}\right)^{\frac{i \omega}{3 \kappa}} \delta^{-\frac{2 i \omega}{3 \kappa}} \psi_{u}(s, \tau)+c_{v} \psi_{v}(s, \tau) .
\end{aligned}
$$


For $\psi_{d}, \psi_{l}$ the matching is analogous. Only the scattering on the geometry may contribute to $c_{v}$. See below.

3.5. Thermality and grey-body factor. We note that the conserved current (3.4) can be rewritten in terms of rescaled variables as follows:

$$
J_{s} \propto \operatorname{Im}\left(i \bar{\omega} \bar{v} \psi^{*} \psi+\left(\bar{c}^{2}-\bar{v}^{2}\right) \psi^{*} \partial_{s} \psi+\frac{1}{3} \delta^{2}\left(\psi^{*} \partial_{s}\left(\left(\frac{h}{h_{0}}\right)^{3} \partial_{s}^{2} \psi\right)-\left(\frac{h}{h_{0}}\right)^{3}\left(\partial_{s} \psi^{*}\right)\left(\partial_{s}^{2} \psi\right)\right)\right) .
$$

We can easily prove that $\frac{\left|J_{s}^{-}\right|}{\left|J_{s}^{+}\right|}=e^{-\beta \omega}$, where $\beta=\frac{2 \pi}{\kappa}$. The grey-body factor can be obtained also in this case, analogously to the cases previously discussed, and the considerations we made for the BEC case and the cases discussed in [1] hold true also for the present case, so we don't discuss them again.

Again, the scattering involving also the mode $v$, in the present framework, is of a different nature with respect to the one occurring for the production process of the Hawking mode $u$, and is associated with the scattering of Hawking modes on the background geometry provided by the reduced equation with $\delta=0$. We expect that also in this case the result is model-dependent, i.e. it should depend strongly on the particular profiles one chooses for the different background fields involved. Furthermore, the metric involved in the model studied in [37] and taken into account herein corresponds to the case $\rho(x)=c^{2}(x)$ of [34, and the corresponding metric (2D part) is

$$
d s^{2}=c(x)\left(c(x)^{2} d t^{2}-(d x-v(x) d t)^{2}\right),
$$

so that the equation one obtains when dispersion is neglected is just 34

$$
\left[\left(\partial_{t}+\partial_{x} v\right)\left(\partial_{t}+v \partial_{x}\right)-\partial_{x} c^{2} \partial_{x}\right] \phi=0
$$

which amounts to the reduced equation. We do not calculate the backscattering contribution to the grey-body factor, instead we limit ourselves to notice that for $c(x)=$ const., as in the case of the subluminal Corley model [1, one would obtain $\Gamma=1$ up to higher order corrections, and then $R=0$. A truncation of the spectrum is to be meant for $\omega>\omega_{\max }$ also in this case, as well known. $\omega_{\max }$ has the same meaning as e.g. in the BEC case.

We cannot claim that the present approach is the solution to the problem at hand, as a more detailed analysis of the experimental situations and of numerical simulations would be needed. Furthermore, we stress that we have not tried to take into account the so-called subcritical case [35, 36, 37, which requires a further analysis to be dealt with.

\section{Conclusions}

We have first taken into account the BEC case and, under the assumption of small (but never vanishing) healing length we have found a scheme allowing to determine in a fully analytical way both the thermality and the grey-body factor of analogous Hawking radiation from BEC analogous black holes. Our analysis confirms that the master equation introduced in [1] is actually effective also in the present case. We have also proposed an improvement in the near horizon analysis of the superluminal case, which is different from previous proposals like e.g. 14, 28. In the second part of the paper, we have taken into account the the problem of surface gravity waves. Thermality is again found in a simple way, and a scheme for the calculation of the grey-body factor is provided. Albeit the present framework provides a very interesting analytical picture of the Hawking effect in analogue gravity, it is by no means exhaustive and further analysis is required to delve into e.g the very low frequency regime in the case of surface waves.

\section{ACKNOWLEDGEMENTS}

We thank Germain Rousseaux for sending us a list of references for completing our bibliography on water experiments and for sending us Ref. [13. A.V. was partially supported by MIUR-PRIN contract 2017CC72MK_003.

\section{Appendix A. Coefficients for the BEC equations}

\section{A.1. Equations for $\phi$.}


A.1.1. Coefficients of the simplified equation. The coefficients which appears in 2.13,

$$
\left[\alpha_{4}(x) \partial_{x}^{4}+\alpha_{2}(x) \partial_{x}^{2}+\alpha_{1}(x) \partial_{x}+\alpha_{0}(x)\right] \zeta(x)=0,
$$

where $\phi(x)=c(x) \sqrt{v(x)} \zeta(x)$, are

$$
\begin{aligned}
& \alpha_{4}=\frac{\hbar^{2}}{4 m^{2} c^{2}(x)}, \\
& \alpha_{3}=-\frac{\hbar^{2} c^{\prime}(x)}{m^{2} c^{3}(x)}-\frac{\hbar^{2} v^{\prime}(x)}{2 m^{2} c^{2}(x) v(x)}, \\
& \alpha_{2}=\frac{\hbar^{2} c^{\prime \prime}(x)}{m^{2} c^{3}(x)}-\frac{3 \hbar^{2} c^{\prime}(x)^{2}}{2 m^{2} c^{4}(x)}-\frac{i \hbar v(x) c^{\prime}(x)}{m c^{3}(x)}+\frac{\hbar^{2} v^{\prime \prime}(x)}{4 m^{2} c^{2}(x) v(x)} \\
& -\frac{3 \hbar^{2} v^{\prime}(x)^{2}}{8 m^{2} c^{2}(x) v^{2}(x)}+\frac{i \hbar v^{\prime}(x)}{m c^{2}(x)}+\frac{v^{2}(x)}{c^{2}(x)}-1, \\
& \alpha_{1}=\frac{\hbar^{2} c^{(3)}(x)}{m^{2} c^{3}(x)}-\frac{i \hbar v(x) c^{\prime \prime}(x)}{m c^{3}(x)}+\frac{3 \hbar^{2} c^{\prime}(x)^{3}}{m^{2} c(x)^{5}}-\frac{i \hbar c^{\prime}(x) v^{\prime}(x)}{m c^{3}(x)}+\frac{i \hbar v(x) c^{\prime}(x)^{2}}{m c^{4}(x)}-\frac{2 \omega \hbar c^{\prime}(x)}{m c^{3}(x)}-\frac{2 c^{\prime}(x)}{c(x)}-\frac{4 \hbar^{2} c^{\prime}(x) c^{\prime \prime}(x)}{m^{2} c^{4}(x)} \\
& +\frac{\hbar^{2} v^{(3)}(x)}{4 m^{2} c^{2}(x) v(x)}+\frac{3 \hbar^{2} v^{\prime}(x)^{3}}{4 m^{2} c^{2}(x) v^{3}(x)}-\frac{\hbar^{2} v^{\prime}(x) v^{\prime \prime}(x)}{m^{2} c^{2}(x) v^{2}(x)}+\frac{i \hbar v^{\prime \prime}(x)}{m c^{2}(x)}+\frac{2 v(x) v^{\prime}(x)}{c^{2}(x)}-\frac{2 i \omega v(x)}{c^{2}(x)}, \\
& \alpha_{0}=\frac{\hbar^{2} c^{(4)}(x)}{4 m^{2} c^{3}(x)}-\frac{\hbar^{2} c^{\prime \prime}(x)^{2}}{2 m^{2} c^{4}(x)}+\frac{i \hbar c^{\prime \prime}(x) v^{\prime}(x)}{2 m c^{3}(x)}-\frac{\omega \hbar c^{\prime \prime}(x)}{m c^{3}(x)}+\frac{v^{2}(x) c^{\prime \prime}(x)}{c^{3}(x)}-\frac{c^{\prime \prime}(x)}{c(x)}-\frac{\hbar^{2} v^{(3)}(x) c^{\prime}(x)}{4 m^{2} c^{3}(x) v(x)}+\frac{\hbar^{2} c^{\prime}(x)^{2} v^{\prime \prime}(x)}{4 m^{2} c^{4}(x) v(x)} \\
& -\frac{3 \hbar^{2} c^{\prime}(x) v^{\prime}(x)^{3}}{4 m^{2} c^{3}(x) v^{3}(x)}-\frac{3 \hbar^{2} c^{\prime}(x)^{2} v^{\prime}(x)^{2}}{8 m^{2} c^{4}(x) v^{2}(x)}+\frac{\hbar^{2} c^{\prime}(x) v^{\prime}(x) v^{\prime \prime}(x)}{m^{2} c^{3}(x) v^{2}(x)}+\frac{i \hbar c^{\prime}(x) v^{\prime \prime}(x)}{2 m c^{3}(x)}-\frac{3 i \hbar c^{\prime}(x) v^{\prime}(x)^{2}}{4 m c^{3}(x) v(x)}-\frac{3 i \hbar c^{\prime}(x)^{2} v^{\prime}(x)}{2 m c^{4}(x)} \\
& +\frac{3 i \hbar v(x) c^{\prime}(x)^{3}}{m c(x)^{5}}+\frac{\omega \hbar c^{\prime}(x)^{2}}{m c^{4}(x)}+\frac{v(x) c^{\prime}(x) v^{\prime}(x)}{c^{3}(x)}-\frac{2 v^{2}(x) c^{\prime}(x)^{2}}{c^{4}(x)}-\frac{\hbar^{2} c^{(3)}(x) c^{\prime}(x)}{m^{2} c^{4}(x)}+\frac{3 \hbar^{2} c^{\prime}(x)^{2} c^{\prime \prime}(x)}{2 m^{2} c(x)^{5}} \\
& -\frac{2 i \hbar v(x) c^{\prime}(x) c^{\prime \prime}(x)}{m c^{4}(x)}+\frac{\hbar^{2} v^{(4)}(x)}{8 m^{2} c^{2}(x) v(x)}-\frac{7 \hbar^{2} v^{\prime \prime}(x)^{2}}{16 m^{2} c^{2}(x) v^{2}(x)}-\frac{63 \hbar^{2} v^{\prime}(x)^{4}}{64 m^{2} c^{2}(x) v^{4}(x)}-\frac{5 \hbar^{2} v^{(3)}(x) v^{\prime}(x)}{8 m^{2} c^{2}(x) v^{2}(x)} \\
& +\frac{31 \hbar^{2} v^{\prime}(x)^{2} v^{\prime \prime}(x)}{16 m^{2} c^{2}(x) v^{3}(x)}-\frac{3 i \hbar v^{\prime}(x)^{3}}{4 m c^{2}(x) v^{2}(x)}+\frac{i \hbar v^{\prime}(x) v^{\prime \prime}(x)}{m c^{2}(x) v(x)}+\frac{v(x) v^{\prime \prime}(x)}{2 c^{2}(x)}-\frac{i \omega v^{\prime}(x)}{c^{2}(x)}+\frac{v^{\prime}(x)^{2}}{4 c^{2}(x)} \\
& -\frac{\omega^{2}}{c^{2}(x)}-\frac{v^{\prime \prime}(x)}{2 v(x)}+\frac{3 v^{\prime}(x)^{2}}{4 v^{2}(x)} \text {. }
\end{aligned}
$$

A.1.2. Coefficients expanded in powers of the healing length. The coefficients which appears in 2.18),

$$
\left[\bar{\xi}^{2} \partial_{x}^{4}-\left(\beta_{2}(x, \bar{\xi}) \partial_{x}^{2}+\beta_{1}(x, \bar{\xi}) \partial_{x}+\beta_{0}(x, \bar{\xi})\right)\right] \zeta(x)=0
$$

are

$$
\begin{aligned}
\beta_{2}=\frac{2 c^{2}(x)}{\bar{c}^{2}} & {\left[\frac{v^{2}(x)}{c^{2}(x)}-1+\left(-\frac{i \sqrt{2} \bar{c} v(x) c^{\prime}(x)}{c^{3}(x)}+\frac{i \sqrt{2} \bar{c} v^{\prime}(x)}{c^{2}(x)}\right) \bar{\xi}+\left(\frac{\bar{c}^{2} v^{\prime \prime}(x)}{2 c^{2}(x) v(x)}-\frac{3 \bar{c}^{2} v^{\prime}(x)^{2}}{4 c^{2}(x) v^{2}(x)}+\frac{2 \bar{c}^{2} c^{\prime \prime}(x)}{c^{3}(x)}-\frac{3 \bar{c}^{2} c^{\prime}(x)^{2}}{c^{4}(x)}\right) \bar{\xi}^{2}\right] } \\
\beta_{1}= & \frac{2 c^{2}(x)}{\bar{c}^{2}}\left[-\frac{2 i \omega v(x)}{c^{2}(x)}+\frac{2 v(x) v^{\prime}(x)}{c^{2}(x)}-\frac{2 c^{\prime}(x)}{c(x)}\right. \\
& +\left(-\frac{i \sqrt{2} \bar{c} v(x) c^{\prime \prime}(x)}{c^{3}(x)}-\frac{i \sqrt{2} \bar{c} c^{\prime}(x) v^{\prime}(x)}{c^{3}(x)}+\frac{i \sqrt{2} \bar{c} v(x) c^{\prime}(x)^{2}}{c^{4}(x)}-\frac{2 \sqrt{2} \bar{c} \omega c^{\prime}(x)}{c^{3}(x)}++\frac{i \sqrt{2} \bar{c} v^{\prime \prime}(x)}{c^{2}(x)}\right) \bar{\xi} \\
& \left.+\left(\frac{\bar{c}^{2} v^{(3)}(x)}{2 c^{2}(x) v(x)}+\frac{3 \bar{c}^{2} v^{\prime}(x)^{3}}{2 c^{2}(x) v^{3}(x)}-\frac{2 \bar{c}^{2} v^{\prime}(x) v^{\prime \prime}(x)}{c^{2}(x) v^{2}(x)}+\frac{2 \bar{c}^{2} c^{(3)}(x)}{c^{3}(x)}-\frac{8 \bar{c}^{2} c^{\prime}(x) c^{\prime \prime}(x)}{c^{4}(x)}+\frac{6 \bar{c}^{2} c^{\prime}(x)^{3}}{c(x)^{5}}\right) \bar{\xi}^{2}\right]
\end{aligned}
$$




$$
\begin{aligned}
\beta_{0}= & \frac{2 c^{2}(x)}{\bar{c}^{2}}\left[\frac{v(x) v^{\prime \prime}(x)}{2 c^{2}(x)}-\frac{i \omega v^{\prime}(x)}{c^{2}(x)}+\frac{v^{\prime}(x)^{2}}{4 c^{2}(x)}-\frac{\omega^{2}}{c^{2}(x)}-\frac{v^{\prime \prime}(x)}{2 v(x)}+\frac{3 v^{\prime}(x)^{2}}{4 v^{2}(x)}+\frac{v(x) c^{\prime}(x) v^{\prime}(x)}{c^{3}(x)}-\frac{2 v^{2}(x) c^{\prime}(x)^{2}}{c^{4}(x)}\right. \\
& +\frac{v^{2}(x) c^{\prime \prime}(x)}{c^{3}(x)}-\frac{c^{\prime \prime}(x)}{c(x)}+\left(\frac{i \bar{c} c^{\prime \prime}(x) v^{\prime}(x)}{\sqrt{2} c^{3}(x)}-\frac{\sqrt{2} \bar{c} \omega c^{\prime \prime}(x)}{c^{3}(x)}+\frac{i \bar{c} c^{\prime}(x) v^{\prime \prime}(x)}{\sqrt{2} c^{3}(x)}-\frac{3 i \bar{c} c^{\prime}(x) v^{\prime}(x)^{2}}{2 \sqrt{2} c^{3}(x) v(x)}-\frac{3 i \bar{c} c^{\prime}(x)^{2} v^{\prime}(x)}{\sqrt{2} c^{4}(x)}\right. \\
& \left.+\frac{3 i \sqrt{2} \bar{c} v(x) c^{\prime}(x)^{3}}{c(x)^{5}}+\frac{\sqrt{2} \bar{c} \omega c^{\prime}(x)^{2}}{c^{4}(x)}-\frac{2 i \sqrt{2} \bar{c} v(x) c^{\prime}(x) c^{\prime \prime}(x)}{c^{4}(x)}-\frac{3 i \bar{c} v^{\prime}(x)^{3}}{2 \sqrt{2} c^{2}(x) v^{2}(x)}+\frac{i \sqrt{2} \bar{c} v^{\prime}(x) v^{\prime \prime}(x)}{c^{2}(x) v(x)}\right) \bar{\xi} \\
& +\left(\frac{\bar{c}^{2} v^{(4)}(x)}{4 c^{2}(x) v(x)}-\frac{7 \bar{c}^{2} v^{\prime \prime}(x)^{2}}{8 c^{2}(x) v^{2}(x)}-\frac{63 \bar{c}^{2} v^{\prime}(x)^{4}}{32 c^{2}(x) v^{4}(x)}-\frac{5 \bar{c}^{2} v^{(3)}(x) v^{\prime}(x)}{4 c^{2}(x) v^{2}(x)}+\frac{31 \bar{c}^{2} v^{\prime}(x)^{2} v^{\prime \prime}(x)}{8 c^{2}(x) v^{3}(x)}-\frac{\bar{c}^{2} c^{\prime \prime}(x)^{2}}{c^{4}(x)}\right. \\
& +\frac{\bar{c}^{2} c^{(4)}(x)}{2 c^{3}(x)}-\frac{\bar{c}^{2} v^{(3)}(x) c^{\prime}(x)}{2 c^{3}(x) v(x)}+\frac{\bar{c}^{2} c^{\prime}(x)^{2} v^{\prime \prime}(x)}{2 c^{4}(x) v(x)}-\frac{3 \bar{c}^{2} c^{\prime}(x) v^{\prime}(x)^{3}}{2 c^{3}(x) v^{3}(x)}-\frac{3 \bar{c}^{2} c^{\prime}(x)^{2} v^{\prime}(x)^{2}}{4 c^{4}(x) v^{2}(x)}+\frac{2 \bar{c}^{2} c^{\prime}(x) v^{\prime}(x) v^{\prime \prime}(x)}{c^{3}(x) v^{2}(x)} \\
& \left.\left.+\frac{3 \bar{c}^{2} c^{\prime}(x)^{2} c^{\prime \prime}(x)}{c(x)^{5}}-\frac{2 \bar{c}^{2} c^{(3)}(x) c^{\prime}(x)}{c^{4}(x)}\right) \bar{\xi}^{2}\right] .
\end{aligned}
$$

\section{A.2. Equations for $\varphi$.}

A.2.1. Coefficients of the simplified equation. The coefficients which appear in the equation

$$
\left[\gamma_{4}(x) \partial_{x}^{4}+\gamma_{2}(x) \partial_{x}^{2}+\gamma_{1}(x) \partial_{x}+\gamma_{0}(x)\right] \eta(x)=0
$$

where $\varphi(x)=c(x) \sqrt{v(x)} \eta(x)$, are

$$
\begin{aligned}
& \gamma_{4}=\frac{\hbar^{2}}{4 m^{2} c^{2}(x)}, \\
& \gamma_{2}=\frac{\hbar^{2} c^{\prime \prime}(x)}{m^{2} c^{3}(x)}-\frac{3 \hbar^{2} c^{\prime}(x)^{2}}{2 m^{2} c^{4}(x)}+\frac{i \hbar v(x) c^{\prime}(x)}{m c^{3}(x)}+\frac{\hbar^{2} v^{\prime \prime}(x)}{4 m^{2} c^{2}(x) v(x)}-\frac{3 \hbar^{2} v^{\prime}(x)^{2}}{8 m^{2} c^{2}(x) v^{2}(x)}-\frac{i \hbar v^{\prime}(x)}{m c^{2}(x)}+\frac{v^{2}(x)}{c^{2}(x)}-1, \\
& \gamma_{1}=\frac{\hbar^{2} c^{(3)}(x)}{m^{2} c^{3}(x)}+\frac{i \hbar v(x) c^{\prime \prime}(x)}{m c^{3}(x)}+\frac{3 \hbar^{2} c^{\prime}(x)^{3}}{m^{2} c(x)^{5}}+\frac{i \hbar c^{\prime}(x) v^{\prime}(x)}{m c^{3}(x)}-\frac{i \hbar v(x) c^{\prime}(x)^{2}}{m c^{4}(x)}+\frac{2 \omega \hbar c^{\prime}(x)}{m c^{3}(x)}-\frac{2 c^{\prime}(x)}{c(x)} \\
& -\frac{4 \hbar^{2} c^{\prime}(x) c^{\prime \prime}(x)}{m^{2} c^{4}(x)}+\frac{\hbar^{2} v^{(3)}(x)}{4 m^{2} c^{2}(x) v(x)}+\frac{3 \hbar^{2} v^{\prime}(x)^{3}}{4 m^{2} c^{2}(x) v^{3}(x)}-\frac{\hbar^{2} v^{\prime}(x) v^{\prime \prime}(x)}{m^{2} c^{2}(x) v^{2}(x)}-\frac{i \hbar v^{\prime \prime}(x)}{m c^{2}(x)}+\frac{2 v(x) v^{\prime}(x)}{c^{2}(x)}-\frac{2 i \omega v(x)}{c^{2}(x)}, \\
& \gamma_{0}=\frac{\hbar^{2} c^{(4)}(x)}{4 m^{2} c^{3}(x)}-\frac{\hbar^{2} c^{\prime \prime}(x)^{2}}{2 m^{2} c^{4}(x)}-\frac{i \hbar c^{\prime \prime}(x) v^{\prime}(x)}{2 m c^{3}(x)}+\frac{\omega \hbar c^{\prime \prime}(x)}{m c^{3}(x)}+\frac{v^{2}(x) c^{\prime \prime}(x)}{c^{3}(x)}-\frac{c^{\prime \prime}(x)}{c(x)}-\frac{\hbar^{2} v^{(3)}(x) c^{\prime}(x)}{4 m^{2} c^{3}(x) v(x)}+\frac{\hbar^{2} c^{\prime}(x)^{2} v^{\prime \prime}(x)}{4 m^{2} c^{4}(x) v(x)} \\
& -\frac{3 \hbar^{2} c^{\prime}(x) v^{\prime}(x)^{3}}{4 m^{2} c^{3}(x) v^{3}(x)}-\frac{3 \hbar^{2} c^{\prime}(x)^{2} v^{\prime}(x)^{2}}{8 m^{2} c^{4}(x) v^{2}(x)}+\frac{\hbar^{2} c^{\prime}(x) v^{\prime}(x) v^{\prime \prime}(x)}{m^{2} c^{3}(x) v^{2}(x)}-\frac{i \hbar c^{\prime}(x) v^{\prime \prime}(x)}{2 m c^{3}(x)}+\frac{3 i \hbar c^{\prime}(x) v^{\prime}(x)^{2}}{4 m c^{3}(x) v(x)}+\frac{3 i \hbar c^{\prime}(x)^{2} v^{\prime}(x)}{2 m c^{4}(x)} \\
& -\frac{3 i \hbar v(x) c^{\prime}(x)^{3}}{m c(x)^{5}}-\frac{\omega \hbar c^{\prime}(x)^{2}}{m c^{4}(x)}+\frac{v(x) c^{\prime}(x) v^{\prime}(x)}{c^{3}(x)}-\frac{2 v^{2}(x) c^{\prime}(x)^{2}}{c^{4}(x)}-\frac{\hbar^{2} c^{(3)}(x) c^{\prime}(x)}{m^{2} c^{4}(x)}+\frac{3 \hbar^{2} c^{\prime}(x)^{2} c^{\prime \prime}(x)}{2 m^{2} c(x)^{5}} \\
& +\frac{2 i \hbar v(x) c^{\prime}(x) c^{\prime \prime}(x)}{m c^{4}(x)}+\frac{\hbar^{2} v^{(4)}(x)}{8 m^{2} c^{2}(x) v(x)}-\frac{7 \hbar^{2} v^{\prime \prime}(x)^{2}}{16 m^{2} c^{2}(x) v^{2}(x)}-\frac{63 \hbar^{2} v^{\prime}(x)^{4}}{64 m^{2} c^{2}(x) v^{4}(x)}-\frac{5 \hbar^{2} v^{(3)}(x) v^{\prime}(x)}{8 m^{2} c^{2}(x) v^{2}(x)} \\
& +\frac{31 \hbar^{2} v^{\prime}(x)^{2} v^{\prime \prime}(x)}{16 m^{2} c^{2}(x) v^{3}(x)}+\frac{3 i \hbar v^{\prime}(x)^{3}}{4 m c^{2}(x) v^{2}(x)}-\frac{i \hbar v^{\prime}(x) v^{\prime \prime}(x)}{m c^{2}(x) v(x)}+\frac{v(x) v^{\prime \prime}(x)}{2 c^{2}(x)}-\frac{i \omega v^{\prime}(x)}{c^{2}(x)}+\frac{v^{\prime}(x)^{2}}{4 c^{2}(x)}-\frac{\omega^{2}}{c^{2}(x)}-\frac{v^{\prime \prime}(x)}{2 v(x)}+\frac{3 v^{\prime}(x)^{2}}{4 v^{2}(x)} .
\end{aligned}
$$

A.2.2. Coefficients of the simplified equation with healing length. The coefficients which appear in the equation

$$
\left[\bar{\xi}^{2} \partial_{x}^{4}-\left(\delta_{2}(x, \bar{\xi}) \partial_{x}^{2}+\delta_{1}(x, \bar{\xi}) \partial_{x}+\delta_{0}(x, \bar{\xi})\right)\right] \eta(x)=0,
$$

are

$$
\delta_{2}=\frac{2 c^{2}(x)}{\bar{c}^{2}}\left[\frac{v^{2}(x)}{c^{2}(x)}-1+\left(\frac{i \sqrt{2} \bar{c} v(x) c^{\prime}(x)}{c^{3}(x)}-\frac{i \sqrt{2} \bar{c} v^{\prime}(x)}{c^{2}(x)}\right) \bar{\xi}+\left(\frac{\bar{c}^{2} v^{\prime \prime}(x)}{2 c^{2}(x) v(x)}-\frac{3 \bar{c}^{2} v^{\prime}(x)^{2}}{4 c^{2}(x) v^{2}(x)}+\frac{2 \bar{c}^{2} c^{\prime \prime}(x)}{c^{3}(x)}-\frac{3 \bar{c}^{2} c^{\prime}(x)^{2}}{c^{4}(x)}\right) \bar{\xi}^{2}\right]
$$




$$
\begin{aligned}
& \delta_{1}=\frac{2 c^{2}(x)}{\bar{c}^{2}}\left[-\frac{2 c^{\prime}(x)}{c(x)}+\frac{2 v(x) v^{\prime}(x)}{c^{2}(x)}-\frac{2 i \omega v(x)}{c^{2}(x)}\right. \\
& +\left(\frac{i \sqrt{2} \bar{c} v(x) c^{\prime \prime}(x)}{c^{3}(x)}+\frac{i \sqrt{2} \bar{c} c^{\prime}(x) v^{\prime}(x)}{c^{3}(x)}-\frac{i \sqrt{2} \bar{c} v(x) c^{\prime}(x)^{2}}{c^{4}(x)}+\frac{2 \sqrt{2} \bar{c} \omega c^{\prime}(x)}{c^{3}(x)}-\frac{i \sqrt{2} \bar{c} v^{\prime \prime}(x)}{c^{2}(x)}\right) \bar{\xi} \\
& \left.+\left(\frac{\bar{c}^{2} v^{(3)}(x)}{2 c^{2}(x) v(x)}+\frac{3 \bar{c}^{2} v^{\prime}(x)^{3}}{2 c^{2}(x) v^{3}(x)}-\frac{2 \bar{c}^{2} v^{\prime}(x) v^{\prime \prime}(x)}{c^{2}(x) v^{2}(x)}+\frac{2 \bar{c}^{2} \bar{c}^{(3)}(x)}{c^{3}(x)}-\frac{8 \bar{c}^{2} c^{\prime}(x) c^{\prime \prime}(x)}{c^{4}(x)}+\frac{6 \bar{c}^{2} c^{\prime}(x)^{3}}{c(x)^{5}}\right) \bar{\xi}^{2}\right], \\
& \delta_{0}=\frac{2 c^{2}(x)}{\bar{c}^{2}}\left[\frac{v^{2}(x) c^{\prime \prime}(x)}{c^{3}(x)}-\frac{c^{\prime \prime}(x)}{c(x)}+\frac{v(x) c^{\prime}(x) v^{\prime}(x)}{c^{3}(x)}-\frac{2 v^{2}(x) c^{\prime}(x)^{2}}{c^{4}(x)}+\frac{v(x) v^{\prime \prime}(x)}{2 c^{2}(x)}\right. \\
& -\frac{i \omega v^{\prime}(x)}{c^{2}(x)}+\frac{v^{\prime}(x)^{2}}{4 c^{2}(x)}-\frac{\omega^{2}}{c^{2}(x)}-\frac{v^{\prime \prime}(x)}{2 v(x)}+\frac{3 v^{\prime}(x)^{2}}{4 v^{2}(x)} \\
& +\left(-\frac{i \bar{c} c^{\prime \prime}(x) v^{\prime}(x)}{\sqrt{2} c^{3}(x)}+\frac{\sqrt{2} \bar{c} \omega c^{\prime \prime}(x)}{c^{3}(x)}-\frac{i \bar{c} c^{\prime}(x) v^{\prime \prime}(x)}{\sqrt{2} c^{3}(x)}+\frac{3 i \bar{c} c^{\prime}(x) v^{\prime}(x)^{2}}{2 \sqrt{2} c^{3}(x) v(x)}+\frac{3 i \bar{c} c^{\prime}(x)^{2} v^{\prime}(x)}{\sqrt{2} c^{4}(x)}\right. \\
& \left.-\frac{3 i \sqrt{2} \bar{c} v(x) c^{\prime}(x)^{3}}{c(x)^{5}}-\frac{\sqrt{2} \bar{c} \omega c^{\prime}(x)^{2}}{c^{4}(x)}+\frac{2 i \sqrt{2} \bar{c} v(x) c^{\prime}(x) c^{\prime \prime}(x)}{c^{4}(x)}+\frac{3 i \bar{c} v^{\prime}(x)^{3}}{2 \sqrt{2} c^{2}(x) v^{2}(x)}-\frac{i \sqrt{2} \bar{c} v^{\prime}(x) v^{\prime \prime}(x)}{c^{2}(x) v(x)}\right) \bar{\xi} \\
& +\left(\frac{\bar{c}^{2} v^{(4)}(x)}{4 c^{2}(x) v(x)}-\frac{7 \bar{c}^{2} v^{\prime \prime}(x)^{2}}{8 c^{2}(x) v^{2}(x)}-\frac{63 \bar{c}^{2} v^{\prime}(x)^{4}}{32 c^{2}(x) v^{4}(x)}-\frac{5 \bar{c}^{2} v^{(3)}(x) v^{\prime}(x)}{4 c^{2}(x) v^{2}(x)}+\frac{31 \bar{c}^{2} v^{\prime}(x)^{2} v^{\prime \prime}(x)}{8 c^{2}(x) v^{3}(x)}-\frac{\bar{c}^{2} c^{\prime \prime}(x)^{2}}{c^{4}(x)}\right. \\
& +\frac{\bar{c}^{2} \bar{c}^{(4)}(x)}{2 c^{3}(x)}-\frac{\bar{c}^{2} v^{(3)}(x) c^{\prime}(x)}{2 c^{3}(x) v(x)}+\frac{\bar{c}^{2} c^{\prime}(x)^{2} v^{\prime \prime}(x)}{2 c^{4}(x) v(x)}-\frac{3 \bar{c}^{2} c^{\prime}(x) v^{\prime}(x)^{3}}{2 c^{3}(x) v^{3}(x)}-\frac{3 \bar{c}^{2} c^{\prime}(x)^{2} v^{\prime}(x)^{2}}{4 c^{4}(x) v^{2}(x)}+\frac{2 \bar{c}^{2} c^{\prime}(x) v^{\prime}(x) v^{\prime \prime}(x)}{c^{3}(x) v^{2}(x)} \\
& \left.\left.+\frac{3 \bar{c}^{2} c^{\prime}(x)^{2} c^{\prime \prime}(x)}{c(x)^{5}}-\frac{2 \bar{c}^{2} \bar{c}^{(3)}(x) c^{\prime}(x)}{c^{4}(x)}\right) \bar{\xi}^{2}\right] \text {. }
\end{aligned}
$$

\section{REFERENCES}

[1] F.Belgiorno, S.L.Cacciatori and A.Viganò, Analogue Hawking effect: a master equation. Preprint (2019).

[2] T.Nishimoto, Kŏdai Math. Sem. Rep. 29, (1978), 233.

[3] T.Nishimoto, Kŏdai Math. Sem. Rep. 24, (1972), 281.

[4] T.Nishimoto, Kŏdai Math. Sem. Rep. 20, (1968), 218.

[5] T.Nishimoto, Kŏdai Math. Sem. Rep. 27, (1976), 128.

[6] G. Rousseaux, C. Mathis, P. Maissa, T. G. Philbin and U. Leonhardt, New J. Phys. 10, 053015 (2008) doi:10.1088/13672630/10/5/053015 arXiv:0711.4767 [gr-qc]].

[7] S. Weinfurtner, E. W. Tedford, M. C. J. Penrice, W. G. Unruh and G. A. Lawrence, Phys. Rev. Lett. 106, 021302 (2011) doi:10.1103/PhysRevLett.106.021302 arXiv:1008.1911 [gr-qc]].

[8] J. Chaline, G. Jannes, P. Maissa and G. Rousseaux, Lect. Notes Phys. 870, 145 (2013) arXiv:1203.2492 [physics.flu-dyn]].

[9] S. Weinfurtner, E. W. Tedford, M. C. J. Penrice, W. G. Unruh and G. A. Lawrence, Lect. Notes Phys. 870, 167 (2013) arXiv:1302.0375 [gr-qc]].

[10] L.-P. Euvé, F. Michel, R. Parentani, T. G. Philbin and G. Rousseaux, Phys. Rev. Lett. 117, no. 12, 121301 (2016) doi:10.1103/PhysRevLett.117.121301 arXiv:1511.08145 [physics.flu-dyn]].

[11] J. Steinhauer, Nature Phys. 10, 864 (2014) doi:10.1038/NPHYS3104 arXiv:1409.6550 [cond-mat.quant-gas]].

[12] J. R. Muñoz de Nova, K. Golubkov, V. I. Kolobov and J. Steinhauer, Nature 569, no. 7758, 688 (2019) doi:10.1038/s41586019-1241-0 arXiv:1809.00913 [gr-qc]].

[13] L. P. Euvè, S. Robertson, N. James, A. Fabbri and G. Rousseaux, Phys. Rev. Lett. 124, no. 14, 141101 (2020) doi:10.1103/PhysRevLett.124.141101 arXiv:1806.05539 [gr-qc]].

[14] S. Corley, Phys. Rev. D, 57, 6280 (1998). hep-th/9710075.

[15] L. J. Garay, J. R. Anglin, J. I. Cirac and P. Zoller, Phys. Rev. Lett. 85, 4643 (2000) doi:10.1103/PhysRevLett.85.4643 gr-qc/0002015.

[16] R. Balbinot, A. Fabbri, S. Fagnocchi, A. Recati and I. Carusotto, Phys. Rev. A 78, 021603 (2008) doi:10.1103/PhysRevA.78.021603 arXiv:0711.4520 [cond-mat.other]].

[17] J. Macher and R. Parentani, Phys. Rev. A 80, 043601 (2009) doi:10.1103/PhysRevA.80.043601 arXiv:0905.3634 [condmat.quant-gas]].

[18] P.-E. Larre, A. Recati, I. Carusotto and N. Pavloff, Phys. Rev. A 85, 013621 (2012) doi:10.1103/PhysRevA.85.013621 arXiv:1110.4464 [cond-mat.quant-gas]].

[19] A. Fabbri and C. Mayoral, Phys. Rev. D 83, 124016 (2011) doi:10.1103/PhysRevD.83.124016 arXiv:1004.4876 [gr-qc]]. 
[20] C. Mayoral, A. Fabbri and M. Rinaldi, Phys. Rev. D 83, 124047 (2011) doi:10.1103/PhysRevD.83.124047 arXiv:1008.2125 [gr-qc]].

[21] P. R. Anderson, R. Balbinot, A. Fabbri and R. Parentani, Phys. Rev. D 87, no. $12, \quad 124018$ (2013) doi:10.1103/PhysRevD.87.124018 arXiv:1301.2081 [gr-qc]].

[22] R. Balbinot, I. Carusotto, A. Fabbri, C. Mayoral and A. Recati, Lect. Notes Phys. 870, 181 (2013) arXiv:1207.2660 [gr-qc]].

[23] P. R. Anderson, R. Balbinot, A. Fabbri and R. Parentani, Phys. Rev. D 90, no. 10, 104044 (2014) doi:10.1103/PhysRevD.90.104044 arXiv:1404.3224 [gr-qc]].

[24] P. R. Anderson, A. Fabbri and R. Balbinot, Phys. Rev. D 91, no. 6, 064061 (2015) doi:10.1103/PhysRevD.91.064061 arXiv:1501.01953 [gr-qc]].

[25] A. Fabbri, R. Balbinot and P. R. Anderson, Phys. Rev. D 93, no. 6, 064046 (2016) doi:10.1103/PhysRevD.93.064046 arXiv:1512.08447 [gr-qc]].

[26] A. Coutant and S. Weinfurtner, Phys. Rev. D 97, no. 2, 025006 (2018) doi:10.1103/PhysRevD.97.025006 arXiv:1707.09664 [gr-qc]].

[27] M.S.P. Eastham, The Asymptotic Solution of Linear Differential Systems: Application of the Levinson Theorem. London Mathematical Society Monographs New Series, Vol. 4. Clarendon Press, 1989.

[28] W.G. Unruh, and R. Schutzhold, Phys. Rev. D 71, 024028 (2005). gr-qc/0408009.

[29] A. Coutant, R. Parentani and S. Finazzi, Phys. Rev. D 85, 024021 (2012) arXiv:1108.1821 [hep-th]].

[30] A. Coutant and R. Parentani, Phys. Rev. D 90, no. 12, 121501 (2014) arXiv:1402.2514 [gr-qc]].

[31] P.D.Miller, Applied Asymptotic Analysis. Graduate Studies in Mathematics, Volume 75. American Mathematical Society, Providence, Rhode Island (2006).

[32] F.Dalfovo, A.Fracchetti, A.Lastri, L.Pitaevskii, and S.Stringari, J. Low Temp. Phys. 104, 367 (1996).

[33] R. Schutzhold and W. G. Unruh, Phys. Rev. D 66, 044019 (2002) doi:10.1103/PhysRevD.66.044019 gr-qc/0205099.

[34] A. Coutant and R. Parentani, Phys. Fluids 26, 044106 (2014) arXiv:1211.2001 [physics.flu-dyn].

[35] L. P. Euvé, F. Michel, R. Parentani and G. Rousseaux, Phys. Rev. D 91, no. 2, 024020 (2015) doi:10.1103/PhysRevD.91.024020 arXiv:1409.3830 [gr-qc]].

[36] S. Robertson, F. Michel and R. Parentani, Phys. Rev. D 93, no. 12, 124060 (2016) doi:10.1103/PhysRevD.93.124060 arXiv:1604.07253 [gr-qc]].

[37] A. Coutant and S. Weinfurtner, Phys. Rev. D 94, no. 6, 064026 (2016) doi:10.1103/PhysRevD.94.064026 arXiv:1603.02746 $[\mathrm{gr}-\mathrm{qc}]]$.

[38] A. Coutant and S. Weinfurtner, Phys. Rev. D 97, no. 2, 025005 (2018) doi:10.1103/PhysRevD.97.025005 arXiv:1707.09651 [gr-qc]].

[39] M. Richartz, A. Prain, S. Liberati and S. Weinfurtner, Phys. Rev. D 91, no. 12, 124018 (2015) doi:10.1103/PhysRevD.91.124018 arXiv:1411.1662 [gr-qc]].

[40] R. S. Johnson, A Modern Introduction to the Mathematical Theory of Water Waves. Cambridge Texts in Applied Mathematics Vol. 19. Cambridge University Press, Cambridge (1997).

${ }^{1}$ Dipartimento di Matematica, Politecnico di Milano, Piazza Leonardo 32, IT-20133 Milano, Italy

${ }^{2}$ INFN Sezione di Milano, via Celoria 16, IT-20133 Milano, Italy

${ }^{3}$ INDAM-GNFM

${ }^{4}$ Department of Science and High Technology, Università dell'Insubria, Via Valleggio 11, iT-22100 Como, Italy

${ }^{5}$ Dipartimento di Fisica, Università degli Studi di Milano, Via Celoria 16, IT-20133 Milano, Italy 